\title{
Checklist of Weeds in University of Port Harcourt and Its Environs
}

\author{
*EKEKE, C; OGAZIE, CA; AGBAGWA, IO
}

\author{
Department of Plant Science and Biotechnology, Faculty of Science \\ University of Port Harcourt, Nigeria \\ *Corresponding Author Email: ekeke.uc@gmail.com
}

\begin{abstract}
We took inventory of the weeds in and around the University of Port Harcourt to determine their family, genus and species compositions and thereby generate their checklist. Three hundred and twenty-two (322) species of weeds belonging to 172 genera and 45 families were identified. Among these weed species, the grasses (Poaceae) having 72 species and 40 genera was the dominant weed identified. This is followed by Fabaceae ( 25 species in 16 genera), Cyperaceae (53 species in 14 genera), Asteraceae ( 15 species in 12 genera) and Commelinaceae ( 20 species in 8 genera) Others include Tiliaceae ( 7 species in 6 genera), Euphorbiaceae (13 species in 7 genera), Amaranthaceae ( 5 species in 4 genera), Rubiaceae (6 species in 5), Convolvulaceae (10 species in 4 genera), Solanaceae ( 8 species in 4 genera), Cucurbitaceae (5 species in 4 genera), Malvaceae (7 species 3 genera) and Verbenaceae (5 species in 3 genera). Anacardiaceae, Asclepiadaceae, Boraginaceae, Crassulaceae, Hydrophyllaceae, Loganiceae, Lythraceae, Melastomataceae, Sapinaceae, Sapindaceae, Selaginellaceae, Sphenocleaceae, Sterculiaceae and Urticaceae with one species in one genus were the least dominant families. These weeds are found in wet or dry land (open fields, cultivated Lands, abandoned fallow and lawns) but predominantly found in open fields. The Perennials (54.98\%) are the dominant weeds followed by annuals $(42.42 \%)$ while bi-annuals $(2.60 \%)$ were the least. The broad leaves were the dominant weeds and constituted $76.47 \%$ of the weeds identified, followed by grasses $14.03 \%$ and sedges $9.50 \%$. This finding, therefore, presents the first comprehensive inventory of weeds in the University of Port Harcourt.
\end{abstract}

\section{DOI: $\underline{\text { https://dx.doi.org/10.4314/jasem.v23i4.2 }}$}

Copyright: Copyright (C) 2019 Ekeke et al. This is an open access article distributed under the Creative Commons Attribution License (CCL), which permits unrestricted use, distribution, and reproduction in any medium, provided the original work is properly cited.

Dates: Received: 30 February 2019; Revised: 27 March 2019; Accepted 12 April 2019

Keywords: weed, Poaceae, Loganiceae, Melastomataceae

Weeds are genetically diverse and many common weed species also have the ability to establish themselves rapidly in the field (Mikulka and Chodová, 2000). This is primarily due to their ability to produce a large number of viable seeds or vegetative tissues such as rhizomes in a single growing season (Shetto et $a l ., 1994)$. In recent times, a considerable increase in infestation of arable land with perennial weeds have been reported (Winkler, 2000). This trend was attributed to the failure of the farmers to carry out cultural practices as well as poor weed control management practices during this process (Mikulka and Chodová, 2000). Weeds compete with crops for water, soil nutrients, light and space and thus reduce crop yields (Sankaran and Mani, 1972; Rao, 1983; Croon et al., 1984; Rain, 1984) and are major causes of low yield in cassava and the main constraint limiting the competitiveness of cassava farmers in the country (Daily Post, 2018). They also contribute to a reduction in maize yield (Shetto et al., 1994; Crammer 1976), rice, wheat, sorghum and millet (Crammer, 1976). West Africa lies in the tropics and sub-humid tropics which are characterized by high temperatures and humid ecosystems, a situation which makes the region conducive to weed growth. Hence, weeds constitute a significant component of the pest complex in African farms, consequently, an important constraint in the agricultural production system (Takim and Amodu, 2013). Identification and proper documentation of the weeds help the farmers to identify the best practice to tackle the different species of weeds. In and around the University of Port Harcourt, there is no comprehensive data on the weeds and presents the checklist of the weed in this area for the first time.

\section{MATERIALS AND METHODS}

The weed enumeration was based on plot-less sampling technique. The sampling of the grasses were done randomly along the roads, foot paths, tracts, open fields, farms, etc without any specific plot or quadrat size (Mueller-Dombois and Ellengerg, 1974). The weeds were identified using the handbook of West African Weeds (Akobundu and Agyakwa, 1998; Akobundu et al., 2017; Hutchinson and Dalziel, 1954; Lowe, 1974; Stanfield and Lowe, 1970). Data on weeds species were collected at monthly intervals for five months during the blooming period (June to October) in 2016 and 2017. 


\section{RESULTS AND DISCUSSION}

A total of 322 weed species belonging to 172 genera and 45 families were identified in and around the University of Port Harcourt (Tables 1 and 2). Based on the number of species, Poaceae family had the maximum species composition of 72 species $(22.36 \%)$ out of 322 weed species identified. It is followed by Cyperaceae 53 (16.46\%), Fabaceae 25 (7.76\%), Asteraceae 15 (4.66\%), Euphorbiaceae 13 (4.04\%), Convolvulaceae 10 (3.11\%), Moraceae 9 (2.80\%), Solanaceae $8(2.48 \%)$, Tiliaceae and Malvaceae 7 $(2.17 \%)$ each, Tiliaceae and Rubiaceae 6 (1.86\%) each, and Amaranthaceae, Cucurbitaceae, Verbenaceae and Vitaceae 5 (1.55\%). The least number of species were in Zingiberaceae, Anacardiaceae, Asclepiadaceae, Boraginaceae,
Crassulaceae, Hydrophyllaceae, Loganiceae, Lythraceae, Melastomataceae, Sapinaceae, Sapindaceae, Selaginellaceae, Sphenocleaceae, Sterculiaceae and Urticaceae (Table 2). Also, members of the family Poaceae with 40 genera $(23.26 \%)$ had the highest number of genera, followed by Fabaceae 16 (9.30\%), Cyperaceae, 14 (8.14\%), Asteraceae 12 (6.97\%), Commelinaceae 8 (4.65\%), Euphorbiaceae $7(4.07 \%)$, Tiliaceae, Rubiaceae and Cucurbitaceae 5 (2.91\%) each, Convolvulaceae, Amaranthaceae and Solanaceae $4(2.33 \%)$ genera each, Malvaceae, Verbenaceae, Acanthaceae, Apocynaceae and Araceae 3 (1.74\%) genera each and Scrophulariaceae, Portulacaeae, Lamiaceae, Passifloraceae, Piperaceae and Sapindaceae 2 (1.16\%) genera each (Table 2).

Table 1: Checklist of weed species identified in the study area

\begin{tabular}{|c|c|c|c|c|}
\hline $\mathrm{S} / \mathrm{N}$ & Name of Weed & Family & Habitat & Lifeform \\
\hline 1 & Asystasia gangetica (Linn.) T. Anders & Acanthaceae & $\mathrm{OF}$ & A \\
\hline 2 & Hypoestes forskalei (Vahl) Soland. Ex Roem. \& Schult. & Acanthaceae & OF & A \\
\hline 3 & Hypoestes sp. & Acanthaceae & $\mathrm{OF}$ & A \\
\hline 4 & Nelsonia canescens (Lam.) Spreng. & Acanthaceae & OF & BA \\
\hline 5 & Alternanthera sessilis (L.) DC. & Amaranthaceae & $\mathrm{OF} / \mathrm{WL}$ & A \\
\hline 6 & Amaranthus spinosus Linn. & Amaranthaceae & $\mathrm{OF} / \mathrm{CL}$ & A \\
\hline 7 & Amaranthus viridis Linn. & Amaranthaceae & $\mathrm{OF} / \mathrm{CL}$ & A \\
\hline 8 & Celosia leptostachya Benth. & Amaranthaceae & $\mathrm{OF}$ & A \\
\hline 9 & Cyathula prostrata (L.) Blume & Amaranthaceae & $\mathrm{OF}$ & A \\
\hline 10 & Gomphrena celosioides Mart. & Amaranthaceae & OF & A \\
\hline 11 & Spondias mombin Linn. & Anacardiaceae & OF & $\mathrm{P}$ \\
\hline 12 & Rauvolfia vomitoria Afzel. & Apocynaceae & OF & $\mathrm{P}$ \\
\hline 13 & Tabernaemontana longiflora Benth. & Apocynaceae & OF & $\mathrm{P}$ \\
\hline 14 & Voacanga africana Stapf & Apocynaceae & $\mathrm{OF}$ & $\mathrm{P}$ \\
\hline 15 & Colocasia esculenta (Linn.) Schott & Araceae & $\mathrm{OF} / \mathrm{CL}$ & $\mathrm{P}$ \\
\hline 16 & Syngonium podophyllum Schott & Araceae & WL/BF & $\mathrm{P}$ \\
\hline 17 & Xanthosoma mafaffa Schott & Araceae & $\mathrm{OF} / \mathrm{CL}$ & $\mathrm{P}$ \\
\hline 18 & Pergularia daemia (Forsk.) Chiov. & Asclepiadaceae & $\mathrm{OF} / \mathrm{BF}$ & $\mathrm{P}$ \\
\hline 19 & Ageratum conyzoides Linn. & Asteraceae & $\mathrm{OF}$ & A \\
\hline 20 & Aspilia Africana (Pers.) C.D. Adams & Asteraceae & OF & A \\
\hline 21 & Chromoleana odorata (L.) R.M. King \& Robinson & Asteraceae & OF & $\mathrm{P}$ \\
\hline 22 & Eclipta alba (L.)Hassk & Asteraceae & $\mathrm{OF} / \mathrm{WL}$ & A \\
\hline 23 & Eleutheranthera ruderalis (Sw.) Sch.Bip. & Asteraceae & $\mathrm{OF}$ & A \\
\hline 24 & Emilia coccinea $($ Sims) G.Don & Asteraceae & OF & A \\
\hline 25 & Emilia praetermissa Milne-Readhead & Asteraceae & $\mathrm{OF}$ & A \\
\hline 26 & Emilia sonchifolia (Linn.) DC. & Asteraceae & OF & A \\
\hline 27 & Ethulia conyzoides & Asteraceae & WL & A \\
\hline 28 & Melanthera scadens (Schum. \& Thonn.) Roberty & Asteraceae & OF & $\mathrm{P}$ \\
\hline 29 & Spilanthes sp. & Asteraceae & $\mathrm{OF}$ & $\mathrm{P}$ \\
\hline 30 & Synedrella nodiflora Gaertn. & Asteraceae & OF & A \\
\hline 31 & Tridax procumbens Linn. & Asteraceae & $\mathrm{OF}$ & BA \\
\hline 32 & Vernonia cinerea (Linn.) Less & Asteraceae & $\mathrm{WL} / \mathrm{BF}$ & $\mathrm{P}$ \\
\hline 33 & Vernonia sp. Linn.f. & Asteraceae & $\mathrm{OF}$ & A \\
\hline 34 & Heliotropium indicum Linn. & Boraginaceae & $\mathrm{OF} / \mathrm{WL}$ & A \\
\hline 35 & Cleome rutidosperma $\mathrm{DC}$. & Cleomaceae & $\mathrm{OF}$ & A \\
\hline 36 & Cleome viscosa $\mathrm{L}$. & Cleomaceae & OF & A \\
\hline 37 & Combretum bracteatum (Laws.) Engl. \& Diels & Combretaceae & $\mathrm{OF} / \mathrm{BF}$ & $\mathrm{P}$ \\
\hline 38 & Combretum platypterum (Welw.) Hutch. \& Dalz. & Combretaceae & $\mathrm{OF} / \mathrm{BF}$ & $\mathrm{P}$ \\
\hline 39 & Combretum racemosum P. Beauv. & Combretaceae & $\mathrm{OF} / \mathrm{BF}$ & $\mathrm{P}$ \\
\hline 40 & Commelina diffusa Burm. $\mathrm{f}$. & Combretaceae & $\mathrm{OF} / \mathrm{BF}$ & $\mathrm{P}$ \\
\hline 41 & Aneilema beninense (P. Beauv.) Kunth & Commelinaceae & $\mathrm{OF} / \mathrm{CL}$ & $\mathrm{A} / \mathrm{P}$ \\
\hline 42 & Aneilema $\mathrm{sp}$ & Commelinaceae & $\mathrm{OF} / \mathrm{CL}$ & $\mathrm{A} / \mathrm{P}$ \\
\hline 43 & Aneilema umbrosum (Vahl) Kunth & Commelinaceae & CL & $\mathrm{A} / \mathrm{P}$ \\
\hline 44 & Aneilema umbrosum subsp. umbrosum J. K. Morton & Commelinaceae & CL & $\mathrm{A} / \mathrm{P}$ \\
\hline 45 & $\begin{array}{l}\text { Aneilema umbrosum subsp. ovato- oblongum (P. } \\
\text { Beauv.) J. K. Morton }\end{array}$ & Commelinaceae & CL & $\mathrm{A} / \mathrm{P}$ \\
\hline
\end{tabular}




\begin{tabular}{|c|c|c|c|}
\hline 46 & Commelina benghalensis var. benghalensis J. K. Morton & Commelinaceae & $\mathrm{OF} / \mathrm{CL}$ \\
\hline 47 & Commelina benghalensis var. hirsuta C. B. Cl. & Commelinaceae & $\mathrm{OF} / \mathrm{CL}$ \\
\hline 48 & Commelina diffusa subsp. diffusa J. K. Morton & Commelinaceae & $\mathrm{OF} / \mathrm{CL}$ \\
\hline 49 & Commelina erecta $\mathrm{L}$. subsp. erecta & Commelinaceae & WL/CL \\
\hline 50 & Commelina erecta Linn. & Commelinaceae & $\mathrm{OF} / \mathrm{CL}$ \\
\hline 51 & Commelina forskalaei $\mathrm{Vahl}$ & Commelinaceae & $\mathrm{OF} / \mathrm{CL}$ \\
\hline 52 & Commelina thomasii Hutch. & Commelinaceae & $\mathrm{OF} / \mathrm{CL}$ \\
\hline 53 & Corchorus trilocularis Linn. & Commelinaceae & $\mathrm{OF}$ \\
\hline 54 & Cyanotis arachnoidea C. B. Cl. & Commelinaceae & $\mathrm{OF}$ \\
\hline 55 & Cyanotis lanata Benth. & Commelinaceae & $\mathrm{OF}$ \\
\hline 56 & Floscopa aquatica Hua & Commelinaceae & CL \\
\hline 57 & Murdannia tenuissima (A. Chev.) Brenan & Commelinaceae & OF \\
\hline 59 & Palisota hirsute (Thunb.) K.Schum. & Commelinaceae & WL \\
\hline 60 & Stanfieldiella imperforata (C. B. Cl.) Brenan & Commelinaceae & CL \\
\hline 61 & Hewittia malabarica (L.) Suresh & Convolvulaceae & OF \\
\hline 62 & Hewittia sublobata Linn. & Convolvulaceae & OF \\
\hline 63 & Ipomoea aquatica Forsk. & Convolvulaceae & WL \\
\hline 64 & Ipomoea asarifolia (Desr.) Roem. \& Schult. & Convolvulaceae & $\mathrm{OF} / \mathrm{WL}$ \\
\hline 65 & Ipomoea cordatotriloba Dennst. & Convolvulaceae & $\mathrm{OF}$ \\
\hline 66 & Ipomoea involucrata $\mathrm{P}$. Beauv. & Convolvulaceae & $\mathrm{OF}$ \\
\hline 67 & Ipomoea mauritiana Jacq. & Convolvulaceae & $\mathrm{OF}$ \\
\hline 68 & Ipomoea quamoclit Linn. & Convolvulaceae & $\mathrm{OF}$ \\
\hline 69 & Lepistemon owariense (P.Beauv.) Hallier f. & Convolvulaceae & OF/WL \\
\hline 70 & Merremia aegyptia (Linn.) Urban & Convolvulaceae & $\mathrm{OF} / \mathrm{WL}$ \\
\hline 71 & Bryophyllum pinnatum (Linn.) Oken & Crassulaceae & $\mathrm{OF}$ \\
\hline 72 & Lagenaria breviflora (Benth.) Roberty & Cucurbitaceae & $\mathrm{OF}$ \\
\hline 73 & Luffa aegyptiaca Mill. & Cucurbitaceae & $\mathrm{OF}$ \\
\hline 74 & Momordica charantia Linn. & Cucurbitaceae & $\mathrm{OF}$ \\
\hline 75 & Zehneria capillacea (Schumach.) C. Jeffrey & Cucurbitaceae & $\mathrm{OF}$ \\
\hline & Croton & Cucurbitaceae & CL \\
\hline 76 & Zehneria scabra (L. f.) Sond. subsp. scabra & Cucurbitaceae & $\mathrm{OF}$ \\
\hline 77 & Bulbostylis barbata (Rottb.) C.B.Cl. & Cyperaceae & $\mathrm{OF} / \mathrm{CL}$ \\
\hline 78 & $\begin{array}{l}\text { Cirpus angolensi C. B. Cl. var. brizaeformis (Hutch.) } \\
\text { Hooper }\end{array}$ & Cyperaceae & WL \\
\hline 79 & Cyperus compressus Linn. & Cyperaceae & $\mathrm{OF} / \mathrm{WL}$ \\
\hline 80 & Cyperus cuspidatus Kunth & Cyperaceae & $\mathrm{OF} / \mathrm{WL}$ \\
\hline 81 & Cyperus dichroöstachyus Hochst. ex A. Rich. & Cyperaceae & OF/WL \\
\hline 82 & Cyperus difformis Linn. & Cyperaceae & $\mathrm{OF} / \mathrm{WL}$ \\
\hline 83 & Cyperus distans Linn.f. & Cyperaceae & $\mathrm{OF} / \mathrm{WL}$ \\
\hline 84 & Cyperus esculentus Linn. & Cyperaceae & $\mathrm{OF} / \mathrm{WL}$ \\
\hline 85 & Cyperus haspan Linn. & Cyperaceae & $\mathrm{OF} / \mathrm{WL}$ \\
\hline 86 & Cyperus iria Linn. & Cyperaceae & $\mathrm{OF} / \mathrm{WL}$ \\
\hline 87 & $\begin{array}{l}\text { Cyperus polystachyos (Rottb.) P. Beauv. var. } \\
\text { polystachyos }\end{array}$ & Cyperaceae & $\mathrm{OF} / \mathrm{WL}$ \\
\hline 88 & Cyperus pustulatus Vahl. & Cyperaceae & $\mathrm{OF} / \mathrm{WL}$ \\
\hline 89 & Cyperus rotundus Linn. & Cyperaceae & OF \\
\hline 90 & Cyperus sp. & Cyperaceae & WL \\
\hline 91 & Cyperus tuberosus Rottb. & Cyperaceae & $\mathrm{OF} / \mathrm{WL}$ \\
\hline 92 & Eleocharis acutangula (Roxb.) J.A. Schultes & Cyperaceae & OF/WL \\
\hline 93 & $\begin{array}{l}\text { Fimbristylis dichotoma var. pluristriata (C.B. Cl.) } \\
\text { Napper }\end{array}$ & Cyperaceae & $\mathrm{OF} / \mathrm{WL}$ \\
\hline 94 & Fimbristylis ferruginea (Linn.) Vahl & Cyperaceae & WL \\
\hline 95 & Fimbristylis hispidula (Vahl) Kunth subsp. hispidula & Cyperaceae & $\mathrm{OF} / \mathrm{WL}$ \\
\hline 96 & Fimbristylis littoralis Gaudet & Cyperaceae & WL \\
\hline 97 & Fuirena ciliaris (Linn.) Roxb. & Cyperaceae & WL \\
\hline 98 & Fuirrena umbellata Rottb. & Cyperaceae & WL \\
\hline 99 & Hypolytrum heteromorphum Nelmes & Cyperaceae & WL \\
\hline 100 & Hypolytrum purpurascens Cherm. & Cyperaceae & WL \\
\hline 101 & Kyllinga brevifolia Rottb. & Cyperaceae & $\mathrm{OF} / \mathrm{WL}$ \\
\hline 102 & Kyllinga bulbosa Beauv. & Cyperaceae & OF/WL \\
\hline 103 & Kyllinga bulbosa P.Beauv. & Cyperaceae & $\mathrm{OF} / \mathrm{WL}$ \\
\hline 104 & Kyllinga erecta Schumach. & Cyperaceae & $\mathrm{OF} / \mathrm{WL}$ \\
\hline 105 & Kyllinga erecta Schumach. var. erecta & Cyperaceae & OF/WL \\
\hline 106 & $\begin{array}{l}\text { Kyllinga erecta Schumach. var. polyphylla (Kunth) } \\
\text { Hooper }\end{array}$ & Cyperaceae & $\mathrm{OF} / \mathrm{WL}$ \\
\hline 107 & Kyllinga erecta Schumacher var. erecta & Cyperaceae & OF/WL \\
\hline 108 & Kylling a erecta var. africana (Kuk.) Hopper & Cyperaceae & $\mathrm{OF} / \mathrm{WL}$ \\
\hline 109 & Kyllinga erecta var. polyphylla (Kunth) Hooper & Cyperaceae & $\mathrm{OF} / \mathrm{WL}$ \\
\hline 110 & Kyllinga nemoralis (Forst.) Dandy ex Hutch. & Cyperaceae & $\mathrm{OF} / \mathrm{WL}$ \\
\hline
\end{tabular}




\begin{tabular}{|c|c|c|c|}
\hline 111 & Kyllinga odorata Vahl & Cyperaceae & OF/WL \\
\hline 112 & Kyllinga peruviana Lam. & Cyperaceae & $\mathrm{OF} / \mathrm{WL}$ \\
\hline 113 & Kyllinga pumila Michx. & Cyperaceae & OF/WL \\
\hline 114 & Kyllinga squamulata Thonn. Ex Vahl & Cyperaceae & OF/WL \\
\hline 115 & Kyllinga tenuifolia Steud. & Cyperaceae & WL \\
\hline 116 & Lipocarpha chinensis (Osbeck) Kern & Cyperaceae & $\mathrm{OF} / \mathrm{WL}$ \\
\hline 117 & Mariscus alternifolius Vahl & Cyperaceae & $\mathrm{OF} / \mathrm{WL}$ \\
\hline 119 & Mariscus dubius (Rottb.) C.E.C. Fischer & Cyperaceae & $\mathrm{OF} / \mathrm{WL}$ \\
\hline 120 & Mariscus flabelliformis Kunth var. flabelliformis & Cyperaceae & OF/WL \\
\hline 121 & Mariscus ligularis (L.) Urban & Cyperaceae & $\mathrm{OF} / \mathrm{WL}$ \\
\hline 122 & Mariscus longibracteatus Cherm. & Cyperaceae & $\mathrm{OF} / \mathrm{WL}$ \\
\hline 123 & Mariscus quarrosus (Linn.) C.B. Cl. & Cyperaceae & $\mathrm{OF} / \mathrm{WL}$ \\
\hline 124 & Mariscus tomaiophyllus (K. Schum.) C.B. Cl. & Cyperaceae & $\mathrm{OF} / \mathrm{WL}$ \\
\hline 125 & Pycreus macrostacyos (Lam.) J.Raynal & Cyperaceae & $\mathrm{OF} / \mathrm{WL}$ \\
\hline 126 & Rhynchospora corymbosa (Linn.) Britt. & Cyperaceae & $\mathrm{OF} / \mathrm{WL}$ \\
\hline 127 & Schoenoplectus senegalensis (Hochst. Ex Steud.) Palla. & Cyperaceae & $\mathrm{OF} / \mathrm{WL}$ \\
\hline 128 & Scleria naumanniana Boeck & Cyperaceae & $\mathrm{OF} / \mathrm{WL}$ \\
\hline 129 & Scleria verrucosa Willd & Cyperaceae & OF/WL \\
\hline 130 & Dioscorea bulbifera & Dioscoreaceae. & $\mathrm{BF}$ \\
\hline 131 & Dioscorea sp. & Dioscoreaceae. & $\mathrm{OF}$ \\
\hline 132 & Acalypha fimbriata Schum. \& Thonn. & Euphorbiaceae & OF \\
\hline 133 & Alchornea cordifolia (Schum \& Thonn) Mull. Arg. & Euphorbiaceae & OF \\
\hline 134 & Alchornea laxiflora (Benth.) Pax \& K. Hoffm & Euphorbiaceae & $\mathrm{OF}$ \\
\hline 135 & Croton hirtus L'Hérit & Euphorbiaceae & $\mathrm{OF}$ \\
\hline 136 & Croton lobatus L. & Euphorbiaceae & OF \\
\hline 137 & Euphorbia heterphylla Linn. & Euphorbiaceae & OF \\
\hline 138 & Euphorbia hirta Linn. & Euphorbiaceae & $\mathrm{OF}$ \\
\hline 139 & Euphorbia hyssopifolia Linn.f. & Euphorbiaceae & $\mathrm{OF}$ \\
\hline 140 & Euphorbia prostrata Ait & Euphorbiaceae & OF \\
\hline 141 & Euphorbia thymifolia Linn. & Euphorbiaceae & OF \\
\hline 142 & $\begin{array}{l}\text { Phyllanthus niruri var. amarus (Schumach. \& Thonn.) } \\
\text { Learndri }\end{array}$ & Euphorbiaceae & $\mathrm{OF}$ \\
\hline 143 & Phyllanthus sp. Linn. & Euphorbiaceae & OF \\
\hline 144 & Phyllanthus urinaria Linn. & Euphorbiaceae & OF \\
\hline 145 & Anthonotha macrpphylla P.Beauv. & Fabaceae & $\mathrm{OF} / \mathrm{BF}$ \\
\hline 146 & Senna alata (L.)Roxb. & Fabaceae & $\mathrm{OF}$ \\
\hline 147 & Senna hirsuta (Linn.) Irwin \& Barneby & Fabaceae & OF \\
\hline 148 & Senna sp. & Fabaceae & $\mathrm{OF}$ \\
\hline 149 & Dialum guineensis Willd. & Fabaceae & $\mathrm{OF} / \mathrm{BF}$ \\
\hline 150 & Aeschynomene indica Linn. & Fabaceae & OF \\
\hline 151 & Mimosa invisa var. inermis Adelb. & Fabaceae & $\mathrm{OF}$ \\
\hline 152 & Mimosa pigra Linn. & Fabaceae & $\mathrm{OF} / \mathrm{WL}$ \\
\hline 153 & Mimosa pudica Linn. & Fabaceae & $\mathrm{OF}$ \\
\hline 154 & Schrankia leptocarpa DC. & Fabaceae & $\mathrm{OF}$ \\
\hline 155 & Baphia nitida Load. & Fabaceae & $\mathrm{OF}$ \\
\hline 156 & Calopogonium mucunoides Desv. & Fabaceae & OF \\
\hline 157 & Centrosema pubescens Benth. & Fabaceae & OF \\
\hline 158 & Crotolaria retusa Linn. & Fabaceae & $\mathrm{OF}$ \\
\hline 159 & Desmodium scorpiurus (Sw.) Desv. & Fabaceae & $\mathrm{OF}$ \\
\hline 160 & Desmodium triflorum (Linn.) DC. & Fabaceae & OF \\
\hline 161 & Dolichos sp. & Fabaceae & $\mathrm{OF}$ \\
\hline 162 & Indigofera $\mathrm{sp}$ & Fabaceae & OF \\
\hline 163 & Indigofera spicata Forsk. & Fabaceae & $\mathrm{OF}$ \\
\hline 164 & Lonchocarpus cyanescens (Schum. \& Thonn.) Benth. & Fabaceae & OF \\
\hline 165 & Millettia aboensis (Hook. f.) Bak. & Fabaceae & $\mathrm{OF}$ \\
\hline 166 & Mucuna cochinchinensis (Lour) A. Chev. & Fabaceae & $\mathrm{OF}$ \\
\hline 167 & Mucuna pruriens (Linn.) DC. var. pruriens & Fabaceae & WL/BF \\
\hline 168 & $\begin{array}{l}\text { Mucuna pruriens var. utilis (Wall. ex Wight) Bak. ex } \\
\text { Burck }\end{array}$ & Fabaceae & $\mathrm{OF}$ \\
\hline 169 & Pueraria phaseoloides (Roxb.) Benth. & Fabaceae & OF \\
\hline 170 & Hydrolea pulustris (Aubl.) Rausch & Hydrophyllaceae & WL \\
\hline 171 & Icacina senegalensis A. Juss. & Icacinaceae & OF \\
\hline 172 & Icacina trichantha Oliv. & Icacinaceae & OF \\
\hline 173 & Platostoma africanum P. Beauv. & Lamiaceae & OF/WL \\
\hline 174 & $\begin{array}{l}\text { Solenostemon monostachyus subsp. monostachyus (P. } \\
\text { Beauv.) Briq. }\end{array}$ & Lamiaceae & $\mathrm{OF}$ \\
\hline 175 & Spigelia anthelmia Linn. & Loganiceae & OF \\
\hline 176 & Ammannia baccifera $\mathrm{L}$. & Lythraceae & WL \\
\hline 177 & Hibiscus asper Hook. f & Malvaceae & $\mathrm{OF}$ \\
\hline 178 & Malvastrum coromandelianum (Linn.) Garcke & Malvaceae & OF \\
\hline
\end{tabular}




\begin{tabular}{|c|c|c|c|}
\hline 179 & Sida acuta Burm.f. & Malvaceae & OF \\
\hline 180 & Sida cordifolia Linn. & Malvaceae & OF \\
\hline 181 & Sida garckeana Polak. & Malvaceae & OF \\
\hline 182 & Sida linifolia Juss ex Cav. & Malvaceae & OF \\
\hline 183 & Sida rhombifolia Linn. & Malvaceae & OF \\
\hline 184 & Heterotis rotundifolia (Sw.) Jac.-Fél. & Melastomataceae & $\mathrm{OF} / \mathrm{WL}$ \\
\hline 185 & Ficus exasperata Vahl. & Moraceae & $\mathrm{OF} / \mathrm{BF}$ \\
\hline 186 & Ficus lyrata & Moraceae & $\mathrm{OF} / \mathrm{BF}$ \\
\hline 187 & Ficus morobensis & Moraceae & $\mathrm{OF} / \mathrm{BF}$ \\
\hline 188 & Ficus ovata & Moraceae & $\mathrm{OF} / \mathrm{BF}$ \\
\hline 189 & Ficus retusa & Moraceae & $\mathrm{OF} / \mathrm{BF}$ \\
\hline 190 & Ficus spp. & Moraceae & $\mathrm{OF} / \mathrm{BF}$ \\
\hline 191 & $\begin{array}{l}\text { Ficus sycomorus sub. sp. gnaphalocarpa (Mig.) C. C. } \\
\text { Berg. }\end{array}$ & Moraceae & $\mathrm{OF} / \mathrm{BF}$ \\
\hline 192 & Ficus triangularis & Moraceae & $\mathrm{OF} / \mathrm{BF}$ \\
\hline 193 & Ficus varirfolia & Moraceae & $\mathrm{OF} / \mathrm{BF}$ \\
\hline 194 & Boerhavia coccinea Mill. & Nyctaginaceae & $\mathrm{OF}$ \\
\hline 195 & Boerhavia diffusa $\mathrm{L}$. & Nyctaginaceae & OF \\
\hline 196 & Nymphaea lotus Linn. & Nymphaeaceae & WL \\
\hline 197 & Nymphaea maculata Schum \& Thonn. & Nymphaeaceae & WL \\
\hline 198 & Ludwigia abyssinica A. Rich. & Onagraceae & WL \\
\hline 199 & Ludwigia decurrens Walt. & Onagraceae & WL \\
\hline 200 & Ludwigia hyssopifolia (G.Don) Exell & Onagraceae & WL \\
\hline 201 & Ludwigia linifolia Vahl & Onagraceae & WL \\
\hline 202 & Adenia gomefora Forsk. & Passifloraceae & $\mathrm{OF}$ \\
\hline 203 & Passiflora foetida Linn. & Passifloraceae & OF \\
\hline 204 & Peperomia pellucida (L.) H. B. \& K & Piperaceae & $\mathrm{OF} / \mathrm{CL}$ \\
\hline 205 & Piperaceae & Piperaceae & WL \\
\hline 206 & Acroceras amplectens Stapf. & Poaceae & WL \\
\hline 207 & Acroceras zizanioides Dandy & Poaceae & WL \\
\hline 208 & Andropogon ascinodis C.B. Cl. & Poaceae & $\mathrm{OF}$ \\
\hline 209 & Andropogon sp. & Poaceae & $\mathrm{OF}$ \\
\hline 210 & Andropogon tectorum Schum. \& Thonn. & Poaceae & OF \\
\hline 211 & Axonopus compressus (Sw.) P. Beauv. & Poaceae & OF \\
\hline 212 & Bambusa vulgaris Wendel. \& & Poaceae & WL/CL \\
\hline 213 & Brachiaria comata (A. Rich.) Stapf & Poaceae & $\mathrm{OF} / \mathrm{WL}$ \\
\hline 214 & Brachiaria deflexa (Shumach.) C.E.Hubbard ex Robyns & Poaceae & $\mathrm{OF}$ \\
\hline 215 & Brachiaria distachyoides Stapf & Poaceae & OF/WL \\
\hline 216 & Brachiaria lata (Shumach.) C. E. Hubbard & Poaceae & $\mathrm{OF}$ \\
\hline 217 & Brachiaria stigmatisata (Mez) Stapf & Poaceae & $\mathrm{OF} / \mathrm{WL}$ \\
\hline 218 & Chloris pilosa Schumach. & Poaceae & OF/WL \\
\hline 219 & Chrysopogon aciculatus (Retz.) Trin. & Poaceae & $\mathrm{OF} / \mathrm{CL}$ \\
\hline 220 & Cymbopogon citratus (DC.) Stapf & Poaceae & $\mathrm{OF} / \mathrm{CL}$ \\
\hline 221 & Cynodon dactylon (Linn.) Pers. & Poaceae & OF/WL \\
\hline 222 & Cynodon nlemfuensis Vanderyst & Poaceae & $\mathrm{OF} / \mathrm{WL}$ \\
\hline 223 & Dactyloctenium aegyptium (Linn.) P.Beauv. & Poaceae & $\mathrm{OF}$ \\
\hline 225 & Digitaria adscendens Henr. & Poaceae & CL \\
\hline 226 & Digitaria argillacea (Hitchc. \& Chase) Fernald & Poaceae & CL \\
\hline 227 & Digitaria debilis (Desf.) Willd. & Poaceae & CL \\
\hline 228 & Digitaria horizontalis Willd. & Poaceae & $\mathrm{OF}$ \\
\hline 229 & Digitaria longiflora (Retz.) Pers. & Poaceae & CL \\
\hline 230 & Echinochloa colona (Linn.) Link & Poaceae & OF/WL \\
\hline 231 & Eleusine indica (L.) Gaertn. & Poaceae & $\mathrm{OF}$ \\
\hline 232 & Eragrostis atrovirens (Desf.) Trin. ex Steud. & Poaceae & $\mathrm{OF} / \mathrm{CL}$ \\
\hline 233 & Eragrostis gangetica Steud. & Poaceae & $\mathrm{OF} / \mathrm{CL}$ \\
\hline 234 & Eragrostis sp. & Poaceae & $\mathrm{OF} / \mathrm{CL}$ \\
\hline 235 & Eragrostis tenella (Linn.) P.Beauv. ex. Roem \& Schult. & Poaceae & $\mathrm{OF} / \mathrm{CL}$ \\
\hline 236 & Euclasta condylotricha (Hochst. ex Steud.) Stapf & Poaceae & $\mathrm{OF} / \mathrm{CL}$ \\
\hline 237 & Guaduella densiflora Pilger & Poaceae & CL \\
\hline 238 & Hackelochloa granularis (Linn.) O.Ktze. & Poaceae & $\mathrm{OF} / \mathrm{CL}$ \\
\hline 239 & Hyparrhenia rufa Stapf & Poaceae & CL \\
\hline 240 & Hyparrhenia involucrata Stapf & Poaceae & CL \\
\hline 241 & $\begin{array}{l}\text { Imperata cylindrica (Linn.) Raeuschel var. africana } \\
\text { (Anderss) C.E. Hubbard }\end{array}$ & Poaceae & $\mathrm{OF} / \mathrm{BF}$ \\
\hline 242 & Leersia hexandra Sw. & Poaceae & WL/CL \\
\hline 243 & Leptochloa caerulescens Steud. & Poaceae & WL/CL \\
\hline 244 & Leptochloa filiformis Beauv. & Poaceae & WL/CL \\
\hline 245 & Oplismenus burmannii (Retz.)P.Beavu. & Poaceae & $\mathrm{OF} / \mathrm{CL}$ \\
\hline 246 & Oryza sativa Linn. & Poaceae & $\mathrm{BF}$ \\
\hline
\end{tabular}


Oxytenanthera abyssinica Munro

Panicum brevifolium Linn.

Panicum brevifolium Linn.

Panicum fluviicola Steud.

Panicum laxum Sw.

Panicum maximum Jacq.

Panicum walense $\mathrm{Mez}$

Paspalum conjugatum Berg.

Paspalum notatum Flüegge

Paspalum scrobiculatum Linn.

Paspalum vaginatum $\mathrm{Sw}$.

Pennisetum pedicellatum Trin.

Pennisetum polystachion (Linn.) Schlt.

Pennisetum purpureum Schumach.

Pennisetum subangustem

Perotis indica (Linn.) O. Ktze

Rhynchelytrum repens (Willd.) C. E. Hubbard

Rottboellia cochinchinensis (Lour.) Clayton

Saccharum officinarum Linn.

Sacciolepis africana Hubb. \& Snowden

Setaria barbata (Lam.) Kunth

Setaria megaphylla (Steud.) Dur. \& Schinz

Setaria pumila (Poir.) Roem. \& Schult

Sorghum arundinaceum (Desv.) Stapf

Sorghum sp.

Sporobolus pyramidalis Beauv.

Sporobolus pyramidalis P. Beauv.

Stenotaphrum secundatum (Walt.) Kuntze

Zea mays L.

Zoysia tenuifolia Willd. ex. Thiele

Talinium triangulare (Jacq.) willd.

Portulaca oleracea Linn.

Portulaca quadrifida Linn.

Diodia sarmentosa $\mathrm{Sw}$.

Mitracapus villosus (Sw.) DC.

Oldenlandia corymbosa Linn.

Oldenlandia herbacea (Linn.) Roxb.

Pentodon pentandrus Schum. \& Thonn.) Vatke

Spermacoce ocymoides Burm.f.

Paullinia pinnata Linn.

Allophylus africanus P. Beauv.

Lindernia cruatacea (Linn.) F.Muelly

Lindernia numulariifolia (D. Don) Wettst.

Lindernia sp.

Scoparia dulcis Linn.

Selaginella myosurus (Sw.) Alston

Datura metel Linn.

Physalis angulata Linn.

Physalis micrantha Linn.

Schwenckia americana Linn.

Solanum macrocarpon Linn.

Solanum nigrum Linn.

Solanum sp.

Solanum torvum Swartz

Sphenoclea zeylanica Gaertn.

Melochia corchorifolia Linn.

Clappertonia ficifolia (Willd.) Decne.

Glyphaea brevis (Spreng.) Monachino

Grewia sp.

Triumfetta cordifolia A.Rich.

Triumfetta rhomboidea Jacq.

Urena lobata Linn.

Pouzolzia guineensis Benth.

Clerodendrum sp. Linn.

Clerodendrum volubile P.Beauv.

Lantana camara Linn.

Stachytarpheta cayennensis (L.C. Rich) Schau.

Stachytarpheta jamaicensis (Linn.) Vahl

Cissus aralioides (Baker) Planch.

Cissus cymosa

\begin{tabular}{|c|c|}
\hline Poaceae & $\mathrm{CL}$ \\
\hline Poaceae & $\mathrm{OF}$ \\
\hline Poaceae & $\mathrm{OF} / \mathrm{CL}$ \\
\hline Poaceae & $\mathrm{OF} / \mathrm{CL}$ \\
\hline Poaceae & OF/WL \\
\hline Poaceae & OF \\
\hline Poaceae & $\mathrm{CL} / \mathrm{BF}$ \\
\hline Poaceae & $\mathrm{WL} / \mathrm{BF}$ \\
\hline Poaceae & OF \\
\hline Poaceae & OF/WL \\
\hline Poaceae & WL \\
\hline Poaceae & $\mathrm{BF}$ \\
\hline Poaceae & $\mathrm{OF}$ \\
\hline Poaceae & $\mathrm{OF}$ \\
\hline Poaceae & OF \\
\hline Poaceae & $\mathrm{CL} / \mathrm{BF}$ \\
\hline Poaceae & CL/BF \\
\hline Poaceae & OF \\
\hline Poaceae & $\mathrm{BF}$ \\
\hline Poaceae & WL \\
\hline Poaceae & OF \\
\hline Poaceae & WL \\
\hline Poaceae & OF \\
\hline Poaceae & $\mathrm{OF} / \mathrm{BF}$ \\
\hline Poaceae & OF \\
\hline Poaceae & $\mathrm{OF}$ \\
\hline Poaceae & $\mathrm{OF}$ \\
\hline Poaceae & OF \\
\hline Poaceae & $\mathrm{BF}$ \\
\hline Poaceae & OF \\
\hline Portulacaeae & OF \\
\hline Portulaceae & $\mathrm{OF}$ \\
\hline Portulaceae & $\mathrm{OF}$ \\
\hline Rubiaceae & OF \\
\hline Rubiaceae & $\mathrm{OF}$ \\
\hline Rubiaceae & $\mathrm{OF} / \mathrm{WL}$ \\
\hline Rubiaceae & $\mathrm{OF}$ \\
\hline Rubiaceae & WL \\
\hline Rubiaceae & $\mathrm{OF}$ \\
\hline Sapindaceae & OF \\
\hline Sapindaceae & $\mathrm{OF} / \mathrm{BF}$ \\
\hline Scrophulariaceae & $\mathrm{OF} / \mathrm{WL}$ \\
\hline Scrophulariaceae & $\mathrm{OF} / \mathrm{WL}$ \\
\hline Scrophulariaceae & OF/WL \\
\hline Scrophulariaceae & $\mathrm{OF}$ \\
\hline Selaginellaceae & WL \\
\hline Solanaceae & $\mathrm{OF}$ \\
\hline Solanaceae & $\mathrm{OF} / \mathrm{CL}$ \\
\hline Solanaceae & $\mathrm{OF} / \mathrm{CL}$ \\
\hline Solanaceae & $\mathrm{OF}$ \\
\hline Solanaceae & OF \\
\hline Solanaceae & $\mathrm{OF}$ \\
\hline Solanaceae & $\mathrm{OF}$ \\
\hline Solanaceae & $\mathrm{OF} / \mathrm{CL}$ \\
\hline Sphenocleaceae & WL \\
\hline Sterculiaceae & $\mathrm{OF} / \mathrm{WL}$ \\
\hline Tiliaceae & WL \\
\hline Tiliaceae & OF \\
\hline Tiliaceae & $\mathrm{OF}$ \\
\hline Tiliaceae & OF \\
\hline Tiliaceae & OF \\
\hline Tiliaceae & OF \\
\hline Urticaceae & $\mathrm{OF}$ \\
\hline Verbenaceae & $\mathrm{OF} / \mathrm{BF}$ \\
\hline Verbenaceae & OF \\
\hline Verbenaceae & OF \\
\hline Verbenaceae & OF \\
\hline Verbenaceae & OF \\
\hline Vitaceae & $\mathrm{OF}$ \\
\hline Vitaceae & $\mathrm{OF} / \mathrm{BF}$ \\
\hline
\end{tabular}

EKEKE, C; OGAZIE, CA; AGBAGWA, IO 


\begin{tabular}{lllll}
318 & Cissus gracills Guill. \& Perr. & Vitaceae & OF & P \\
319 & Cissus simsiana & Vitaceae & OF/BF & P \\
320 & Cissus sp. & Vitaceae & OF & A \\
321 & Costus afer Ker-Gawl. & Zingiberaceae & OF & A \\
322 & Costus dubbius (Afzel.) K.Schum. & Zingiberaceae & WL & P \\
\hline
\end{tabular}

OF - Open field, A- annual, P- perennial, BA- bi-annual, CL- cultivated land, BF- bush fallow, WL- wetland

\begin{tabular}{|c|c|c|c|c|c|c|c|}
\hline \multirow{2}{*}{$\mathbf{S} / \mathbf{N}$} & \multirow{2}{*}{ FAMILY NAME } & \multicolumn{2}{|c|}{ Number of } & \multirow{2}{*}{$\mathbf{S} / \mathbf{N}$} & \multirow{2}{*}{$\begin{array}{l}\text { FAMILY } \\
\text { NAME }\end{array}$} & \multicolumn{2}{|c|}{ Number of } \\
\hline & & Genera & Species & & & Genera & Species \\
\hline 1 & Acanthaceae & 3 & 4 & 24 & Malvaceae & 3 & 7 \\
\hline 2 & Amaranthaceae & 4 & 5 & 25 & Melastomataceae & 1 & 2 \\
\hline 3 & Anacardiaceae & 1 & 1 & 26 & Moraceae & 1 & 9 \\
\hline 4 & Apocynaceae & 3 & 3 & 27 & Nyctaginaceae & 1 & 2 \\
\hline 5 & Araceae & 3 & 3 & 28 & Nymphaeaceae & 1 & 2 \\
\hline 6 & Asclepiadaceae & 1 & 1 & 29 & Onagraceae & 1 & 4 \\
\hline 7 & Asteraceae & 12 & 15 & 30 & Passifloraceae & 2 & 2 \\
\hline 8 & Boraginaceae & 1 & 1 & 31 & Piperaceae & 2 & 2 \\
\hline 9 & Cleomaceae & 1 & 2 & 32 & Poaceae & 40 & 72 \\
\hline 10 & Combretaceae & 1 & 4 & 33 & Portulacaeae & 2 & 3 \\
\hline 11 & Commelinaceae & 8 & 20 & 34 & Rubiaceae & 5 & 6 \\
\hline 12 & Convolvulaceae & 4 & 10 & 35 & Sapindaceae & 2 & 2 \\
\hline 13 & Crassulaceae & 1 & 1 & 36 & Scrophulariaceae & 2 & 4 \\
\hline 14 & Cucurbitaceae & 5 & 5 & 37 & Selaginellaceae & 1 & 1 \\
\hline 15 & Cyperaceae & 14 & 53 & 38 & Solanaceae & 4 & 8 \\
\hline 16 & Dioscoreaceae. & 1 & 2 & 39 & Sphenocleaceae & 1 & 1 \\
\hline 17 & Euphorbiaceae & 7 & 13 & 40 & Sterculiaceae & 1 & 1 \\
\hline 18 & Fabaceae & 16 & 25 & 41 & Tiliaceae & 5 & 6 \\
\hline 19 & Hydrophyllaceae & 1 & 1 & 42 & Urticaceae & 1 & 1 \\
\hline 20 & Icacinaceae & 1 & 2 & 43 & Verbenaceae & 3 & 5 \\
\hline 21 & Lamiaceae & 2 & 2 & 44 & Vitaceae & 1 & 5 \\
\hline 22 & Loganiceae & 1 & 1 & 45 & Zingiberaceae & 1 & 2 \\
\hline 23 & Lythraceae & 1 & 1 & & & & \\
\hline Total & & & & & & 172 & 322 \\
\hline
\end{tabular}

Broad leaves constituted $61.56 \%$ of the total weed species encountered while grasses and sedges constituted $21.88 \%$ and $16.56 \%$ respectively (Figure $1)$.

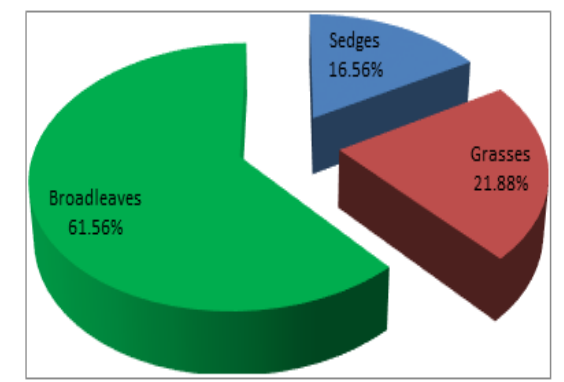

Fig 1: Percentage composition of broad leaves, grasses, and sedges in the study area

Perennials were the dominant weed species identified in the study area and comprised $54.55 \%$ of the total weed species while annuals had $40.13 \%$, annuals/perennials $3.76 \%$ and bi-annuals had $1.57 \%$ (Figure 2). Based on habitat distribution, 133 weed species were found in the open fields only, 65 species in open fields/wetlands, 31 species in only wetland, 28 species in open fields/cultivated lands, 22 in open fields/bush fallow, 14 species in cultivated lands only, 7 species in wetlands/bush fallow and 2 species in bush fallow (Tables 1 and 3).

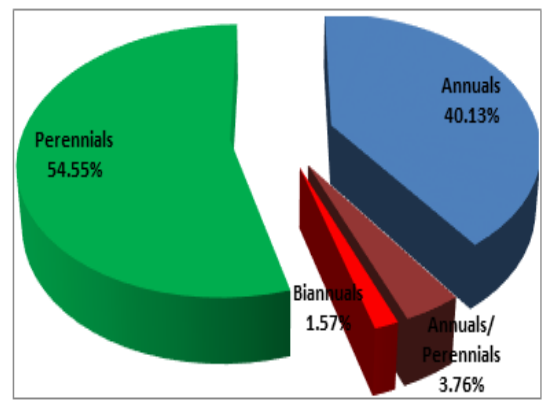

Fig 2: Percentage composition of weeds based on their life form

Table 3: Summary of distribution weeds species based on habitat

\begin{tabular}{lc}
\hline Habitat & No. of species \\
\hline Open fields (OF) & 133 \\
Open fields/Wetlands (OF/WL) & 65 \\
Wetlands (WL) & 31 \\
Open fields/Cultivated lands (OF/CL) & 28 \\
Open fields/Bush fallow (OF/BF) & 22 \\
Cultivated lands (CL) & 14 \\
Wetlands/Bush fallow (WL/BF) & 7 \\
Bush fallow (BF) & 5 \\
Cultivated lands/Bush fallow (CL/BF) & 2 \\
\hline
\end{tabular}

The results from this study showed that broadleaves were the dominant weed in and round the University of Port Harcourt. However, members of the Poaceae family dominated the weed community. Other 
dominant weed families encountered were Cyperaceae, Fabaceae, Asteraceae, Euphorbiaceae, Convolvulaceae, Moraceae, Solanaceae, Tiliaceae, and Malvaceae. Similar observations have been made in sugarcane and legume farms that the broadleaves were the dominant weed species, followed by grasses and sedges (Takim and Amodu, 2013; Lemerle and Murphy, 2000). Studies have also shown that the more diverse the land use system, the more diverse the weed community with less dominant and troublesome species, the more the broadleaves (Cardina et al., 1998). This, however, suggests that there are farming and bush clearing activities within the study area and is evident as most of the weed species are found in open fields, open fields/wetlands, open fields/cultivated lands and open fields/bush fallow. Furthermore, our study showed that perennials and annuals were the predominant weeds identified. In the same vein, perennial weed species have been reported to grow more on less-disturbed and more stable environments. Similarly, our study area is lessdisturbed which inform the dominance of broadleaf weeds and support the findings of Clements et.al. (1996).

Conclusion: The results from this study showed Three hundred and twenty-two (322) species of weeds belonging to 172 genera and 45 families within University of Port Harcourt and its environs with the broadleaves dominating among the weeds. This report represents the first comprehensive inventory on weeds in the University of Port Harcourt and its environs.

\section{REFERENCES}

Cardina, J; Webster, TM; Herms, CP (1998). Longterm tillage and rotation effects on soil seed bank characteristics. Applied Biology 51: 213-220.

Clements, DR; Benoit, DL; Murphy, SD; Swanton, CJ (1996). Tillage effects on weed seed return and seed-bank composition. Weed Science 44: 314322.

Cramer, H (1976). In Weed Control (Eds. Minjas, AN; Jana, RK) Proceedings of a workshop on resource efficient farming methods for Tanzania May 1620, 1983.

Croon, I; Deutsch, J; Temu, AEM (1984). Maize production in Tanzania's southern highlands: current status and recommendations for the future.
Centro International de Mejoramiento de Maiz y Trigo (CIMMYT), Londres, Mexico.

http://dailypost.ng/2018/03/21/nigeria-declaresweeds-major-productivity-drawback-cassavafarming/

Lemerle, D; Murphy, CE (2000). Cultural management methods. In: B.M. Sindel (Ed.), Australian Weed Management Systems pp. 123138.

Lowe, J (1974). The Flora of Nigeria: Sedges (Family Cyperaceae). Ibadan University Press.

Mikulka, J; Chodová, D (2000). Changes in weed societies in the Czech Republic. In: XVth Czech and Slovak Plant Protection Conference, Brno 15: 287-288.

Mueller-Dombois, D; Ellenberg, H (1974). Aims and Methods of Vegetation Ecology. John Wiley \& Sonos Inc., Canada pg. 99-102.

Rain, DK (1984). The constraints to smallholder peasant agricultural production in Mbeya and Mbozi districts, Mbeya region. Report to the Canadian International Development Agency, Hull, Quebec, Canada.

Rao, VS (1983). Principles of weed science. IBH Publishing Company, Oxford.

Sankaran, S. and Mani, V.S. 1972. Effect of weed growth on nutrient uptake and seed yield of sorghum. Indian J. Weed Science, 4.

Shetto, RM; Kwiligwa, EM; Rees, DJ; lay, GJ (1994). Weed management systems based on animaldrawn cultivators for maize production in the Southern Highlands of Tanzania, Soil and Tillage Res. 29(4): 383-395

Stanfield, DP; Lowe, J (1970). The Flora of Nigeria: Grasses. Ibadan University Press.

Takim, FO; Amodu, AA (2013). Quantitative estimate of weeds of sugarcane (Saccharum officinarum L.) crop in Ilorin, Southern Guinea Savanna of Nigeria. Ethio. J. of Env. Stu. and Mgt. 6 (6):611 -619 .

Winkler, J (2000). The changes of species structure of weed communities influence by flood. In: XVth Czech and Slovak Plant Protection Conference, Brno 15: 315-316. 